\title{
A Non-paraxial Model for the Audio Sound behind a Non- baffled Parametric Array Loudspeaker
}

\author{
Jiaxin Zhong ${ }^{1}$, Ray Kirby ${ }^{1}$, Xiaojun Qiu ${ }^{1}$ \\ ${ }^{1}$ Centre for Audio, Acoustics and Vibration, Faculty of Engineering and IT, \\ University of Technology Sydney, NSW 2007, Australia
}

Suggested running title: Audio sound behind a Non-baffled PAL 


\begin{abstract}
It has been reported that audible sounds can be heard behind a parametric array loudspeaker in free field, which cannot be predicted by existing models. A nonparaxial model is developed in this paper for the finite size and disk-shaped parametric source based on the quasilinear approximation and the disk scattering theory. The sounds in both front and back sides are calculated numerically and compared with the existing non-paraxial model for the parametric source installed in an infinitely large baffle. Both simulation and experiment results show that audible audio sound exists in the back side. The mechanism of the phenomenon is explored in the paper.
\end{abstract}




\section{INTRODUCTION}

The parametric acoustic array (PAA) has attracted much attention due to its capability of generating highly directional sound beams without side lobes at low frequencies. ${ }^{1}$ The parametric array loudspeaker (PAL) is an application of PAAs for radiating highly directional audio sounds in air where the carrier wave is ultrasound..$^{2,3}$ The most accurate existing theoretical models of PALs assume that a PAL is placed on an infinitely large baffle, which means that no audio sounds propagate to the back side of the PAL. ${ }^{4}$ However, audible audio sounds in the back side of a PAL were detected in PAL applications because the audio sounds can diffract to the back side ${ }^{5}$. Unfortunately, there is no analytical model for non-baffled PALs at present, which will be developed in this paper for predicting the sound field in the back side of a finite size PAL.

When a PAL radiates two intensive ultrasound (primary) waves at different frequencies, the secondary waves which contain the difference-frequency wave (the audio sound in air) is generated due to the nonlinearity. ${ }^{1}$ The simplest model assumes the ultrasound waves are collimated and fully attenuated in the nearfield and the audio sound is generated by a line array of virtual audio sources along the axis of the PAL. ${ }^{1}$ The directivity of the generated audio sound is very sharp and has no side lobes. The most widely used model for predicting the audio sound is the KhokhlovZabolotskaya-Kuznetsov (KZK) equation which considers the diffraction, absorption and nonlinearity under the paraxial approximation. Many methods can be used to solve the KZK equation analytically or numerically; however, the results are usually only valid in front of the PAL and inside the paraxial region about $20^{\circ}$ from the transducer axis. ${ }^{6}$

A non-paraxial model with better accuracy for baffled PALs was proposed based on the Westervelt's equation and the quasilinear approximation. ${ }^{4}$ By applying the Gaussian beam expansion method to ultrasounds, the computation of audio sounds is reduced to a three-fold integral which can be used to predict the near-field and wide- 
angle far-field sound fields. It is found the low-frequency audio sound is significant in the vicinity of the source because the low directivity of the virtual audio sources cannot be predicted by any models based on the paraxial approximation. The results for a non-baffled PAL are different when the size of a PAL is smaller than the wavelength of audio sounds at low frequencies because the baffled assumption made in Ref. 4 does not hold any more. Furthermore, all of the aforementioned models cannot be used to predict the sound behind a non-baffled PAL.

The finite element method (FEM) and the boundary element method (BEM) are promising techniques for solving linear acoustic problems and they are well built in much commercial simulation software. However, it is almost impossible or very difficult to compute and predict audio sounds generated by non-baffled PALs directly with the existing commercial software because the model is nonlinear. Although the FEM model for the nonlinear sound wave propagation is available, it is timeconsuming and hard to compute the sounds generated by non-baffled PALs in open space. $^{7}$ Therefore, an analytical prediction model for non-baffled PALs is needed.

PALs are usually manufactured in circular or square shapes with small thickness, so it can be treated as a finite size disk or square plate. The sound scattering by a finite size disk can be solved analytically, ${ }^{8}$ so the disk-shaped PAL is considered in this paper. The solution consists of the spheroidal wave functions derived from the oblate spheroidal coordinate system. Although the computation of spheroidal wave functions is complicated, some software or codes are available..$^{9,10}$ In this paper, each virtual audio source generated by ultrasounds of a PAL in space is regarded as a point monopole so that its scattered sound by the finite size disk can be solved. The nonparaxial solution of total audio sounds is exact in both front and back sides of the non-baffled PAL.

\section{THEORY}

The sketch of the model is shown in Fig. 1. Assume a circular PAL with a radius 
of $a$ in free field generates two harmonic ultrasounds with frequencies $f_{1}$ and $f_{2}\left(f_{1}>\right.$ $f_{2}$ ) with the boundary condition on the transducer surface being

$$
v_{z}(x, y, t)=v_{1}(x, y) \mathrm{e}^{\mathrm{j} 1_{1}^{t}}+v_{2}(x, y) \mathrm{e}^{\mathrm{j}{ }_{2} t},
$$

where $\mathrm{j}$ is the complex unit, $v_{z}$ represents the vibration velocity normal to the transducer surface, $v_{i}$ is the amplitude of the vibration velocity, $i=1,2, \omega_{i}=2 \pi f_{i}$ is the angular frequency of the $i$ th primary wave, $(x, y)$ is the transverse coordinate, and the axis $z$ is normal to the transducer surface under the coordinate system $O-x y z$. The solutions of the ultrasounds and audio sound with the frequency $f_{\mathrm{a}}=f_{1}-f_{2}$ are denoted as

$$
\tilde{p}_{i}(\mathbf{r}, t)=p_{i}(\mathbf{r}) \mathrm{e}^{-\mathrm{j} \omega_{i} t}, \quad i=1,2, \mathrm{a},
$$

where the subscript "1", "2", and "a" represent the two ultrasounds and the audio sound respectively, and $\mathbf{r}=(x, y, z)$ is a field location.

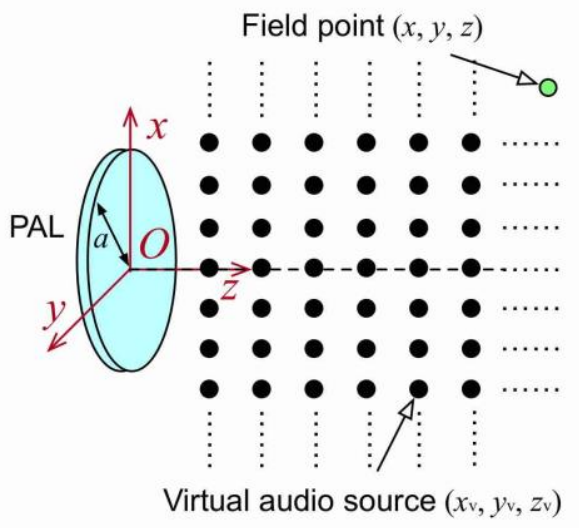

(a)

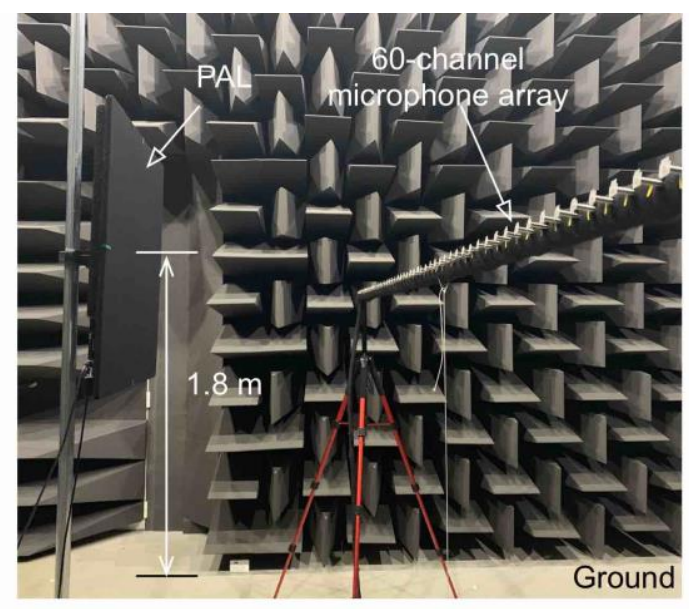

(b)

FIG. 1. (Color Online) A PAL radiating ultrasounds in free field: (a) the sketch of the model; (b) the experiment setup.

Because the ultrasound level generated by a PAL is limited due to safety concerns, the nonlinearity is not very strong and the quasilinear approximation can be used in the derivation. ${ }^{3}$ With the successive method, the sound pressure of the ultrasounds can be expressed by Rayleigh integral as ${ }^{4}$ 


$$
p_{i}(\mathbf{r})=2 \mathrm{j}_{0} \quad i_{i}\left(x_{\mathrm{s}}, y_{\mathrm{s}}\right) \frac{\mathrm{e}^{\mathrm{j} k_{i} d_{\mathrm{s}}}}{4 \pi d_{\mathrm{s}}} \mathrm{d} x_{\mathrm{s}} \mathrm{d} y_{\mathrm{s}}
$$

where $k_{i}={ }_{i} / c_{0}+\mathrm{j}{ }_{i}, \alpha_{i}$ is the sound attenuation coefficient at the frequency $f_{i}{ }^{11} i$ $=1$ and 2, and $d_{\mathrm{s}}=\sqrt{\left(\begin{array}{ll}x & x_{\mathrm{s}}\end{array}\right)^{2}+\left(\begin{array}{ll}y & y_{\mathrm{s}}\end{array}\right)^{2}+z^{2}}$ is the distance between the field point $\mathbf{r}$ and the source point $\left(x_{\mathrm{s}}, y_{\mathrm{s}}, 0\right)$ on the transducer surface.

If the PAL is placed on an infinitely large baffle, the audio sound can be treated as the superposition of the sounds generated by infinitely many virtual sources at $\mathbf{r}_{\mathrm{v}}$ $=\left(x_{\mathrm{v}}, y_{\mathrm{v}}, z_{\mathrm{v}}\right)$ and their image sources at $\left(x_{\mathrm{v}}, y_{\mathrm{v}},-z_{\mathrm{v}}\right)$ which has the source density function of

$$
q\left(\mathbf{r}_{\mathrm{v}}\right)=\frac{\mathrm{j}}{{ }_{0}^{2} c_{0}^{4}} p_{1}\left(\mathbf{r}_{\mathrm{v}}\right) p_{2}^{*}\left(\mathbf{r}_{\mathrm{v}}\right), z_{\mathrm{v}}>0
$$

The audio sound near an infinitely large baffle is then expressed as ${ }^{4}$ (denoted by "baffled model")

$$
p_{\mathrm{a}}(\mathbf{r})=\mathrm{j}_{0} \int_{\mathrm{a}} \int_{0}^{\infty} \int_{\infty}^{\infty} \int_{\infty}^{\infty} q\left(\mathbf{r}_{\mathrm{v}}\right)\left(\frac{\mathrm{e}^{\mathrm{j} k_{\mathrm{a}} d_{\mathrm{v},+}}}{4 \pi d_{\mathrm{v},+}}+\frac{\mathrm{e}^{\mathrm{j} k_{\mathrm{a}} d_{\mathrm{v},}}}{4 \pi d_{\mathrm{v},}}\right) \mathrm{d} x_{\mathrm{v}} \mathrm{d} y_{\mathrm{v}} \mathrm{d} z_{\mathrm{v}}, z>0,
$$

where $k_{\mathrm{a}}={ }_{\mathrm{a}} / c_{0}+\mathrm{j}{ }_{\mathrm{a}}, \alpha_{\mathrm{a}}$ is the sound attenuation coefficient at the frequency $f_{\mathrm{a}}$,

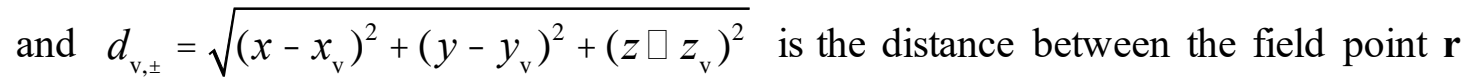
and the virtual source $\left(d_{\mathrm{v},+}\right)$ or its image source $\left(d_{\mathrm{v},-}\right)$.

Although the sound pressure of ultrasounds can be expressed as Eq. (3) for nonbaffled PALs due to the fact that the size of PALs is usually large enough compared with the wavelength of ultrasounds, the PALs usually cannot be considered as infinitely large for the audio sounds. Each virtual audio source with the source density function shown in Eq. (4) is scattered by the disk. The boundary of the disk is assumed to be rigid for audio sounds, so it has ${ }^{12}$

$$
\frac{p_{\mathrm{a}}}{{ }_{=}{ }_{\mathrm{b}}=0}=0
$$

where $\xi$ is the radial oblate spheroidal coordinate in the oblate spheroidal coordinate 
system, $\xi_{\mathrm{b}}$ represents the coordinate of the boundary surface, and the oblate coordinates $(\eta, \xi, \varphi)$ are related to the Cartesian coordinates $(x, y, z)$ by $^{12}$

$$
\left\{\begin{array}{l}
x=a \sqrt{\left(1+{ }^{2}\right)\left(1^{2}\right)} \cos , \\
y=a \sqrt{\left(1+{ }^{2}\right)\left(1^{2}\right)} \sin , \\
z=a .
\end{array}\right.
$$

When the radial coordinate $\xi=\xi_{\mathrm{b}}=0$, the oblate represents an infinitely thin disk with a radius of $a$ centered at $O$ on the plane $x O y$. The total sound pressure at a field point $\mathbf{r}=(\eta, \xi, \varphi)$ radiated by a non-baffled disk-shaped PAL is the superposition of the direct and scattered sounds generated by the virtual audio sources and is expressed as (denoted by "non-baffled model")

$$
p_{\mathrm{a}, \mathrm{t}}(\mathbf{r})=\mathrm{j}_{0} \mathrm{a}^{a^{3}} a_{000}^{2 \pi 1} q\left(\mathbf{r}_{\mathrm{v}}\right) G\left(\mathbf{r}, \mathbf{r}_{\mathrm{v}}\right)\left({\underset{\mathrm{v}}{\mathrm{v}}}^{2}+{ }_{\mathrm{v}}^{2}\right) \mathrm{d}_{\mathrm{v}} \mathrm{d}_{\mathrm{v}} \mathrm{d}_{\mathrm{v}}, z<0 \text { or } z>0,
$$

where the relation $\mathrm{d} x_{\mathrm{v}} \mathrm{d} y_{\mathrm{v}} \mathrm{d} z_{\mathrm{v}}=a^{3}\left(\left(_{\mathrm{v}}^{2}+{ }_{\mathrm{v}}^{2}\right) \mathrm{d}_{\mathrm{v}} \mathrm{d}_{\mathrm{v}}^{\mathrm{d}}{ }_{\mathrm{v}}\right.$ is used with the metric coefficients given by Eq. (15.1.10) in Ref. 9. $G\left(\mathbf{r}, \mathbf{r}_{\mathrm{v}}\right)$ is the Green function in the presence of a rigid disk and can be expressed as ${ }^{12}$

$$
\begin{aligned}
G\left(\mathbf{r}, \mathbf{r}_{\mathrm{v}}\right)=\frac{\mathrm{j} k_{\mathrm{a}}}{2 \pi_{m=0}=m} m m n & \left(\mathrm{j} k_{\mathrm{a}} a, \mathrm{j}_{,} \mathrm{j}_{\mathrm{v}}\right) S_{m n}\left(\mathrm{j} k_{\mathrm{a}} a, j\right) S_{m n}\left(\mathrm{j} k_{\mathrm{a}} a,{ }_{\mathrm{v}}\right) \cos [m( \\
{ }_{m n}\left(\mathrm{j} k_{\mathrm{a}} a, \mathrm{j}_{,} \mathrm{j}_{\mathrm{v}}\right)= & R_{m n}^{(1)}\left(\mathrm{j} k_{\mathrm{a}} a, \mathrm{j}_{<}\right) R_{m n}^{(3)}\left(\mathrm{j} k_{\mathrm{a}} a, \mathrm{j}_{>}\right) \\
& \frac{R_{m n}^{(1)}\left(\mathrm{j}_{\mathrm{a}} a, \mathrm{j}_{\mathrm{b}}\right)}{R_{m n}^{(3)}\left(\mathrm{j}_{\mathrm{a}} a, \mathrm{j}_{\mathrm{b}}\right)} R_{m n}^{(3)}\left(\mathrm{j} k_{\mathrm{a}} a, \mathrm{j}\right) R_{m n}^{(3)}\left(\mathrm{j} k_{\mathrm{a}} a, \mathrm{j}_{\mathrm{v}}\right),
\end{aligned}
$$

where $\mathbf{r}_{\mathrm{v}}=\left(\eta_{\mathrm{v}}, \xi_{\mathrm{v}}, \varphi_{\mathrm{v}}\right)$ is the location of the virtual audio source in the oblate spheroidal coordinate system, $\varepsilon_{m}$ is the Neumann factor i.e. $\varepsilon_{m}=1$ for $m=0$ and $\varepsilon_{m}=$ 2 for $m \neq 0, \xi_{>}=\max \left(\xi, \xi_{\mathrm{v}}\right)$ and $\xi_{<}=\min \left(\xi, \xi_{\mathrm{v}}\right)$. The notations of spheroidal wave functions follow that in Refs. 9 and 10 , where $S_{m n}\left(-\mathrm{j} k_{\mathrm{a}} a, \eta\right)$ is the normalized angular oblate spheroidal wave function, $R_{m n}^{(i)}\left(\mathrm{j} k_{\mathrm{a}} a, \mathrm{j}\right)$ and $R_{m n}^{(i)}\left(\mathrm{j} k_{\mathrm{a}} a, \mathrm{j}\right)$ are the $i$ th kind of the radial oblate spheroidal wave functions and their derivatives with respect to $\xi$, respectively, $i=1,3$. The readers may refer to Refs. 9 and 10 for the computations of these special functions. By substituting Eq. (1) into Eq. (3), the ultrasound fields 
can be calculated and then the audio field can be obtained with Eq. (8) using the source density function in Eq. (4).

It is hard to calculate the audio sound due to the three-fold integral in Eq. (8), the two-fold integral of ultrasounds in Eq. (3), and the two-fold summation of Green function in Eq. (9). When the surface of the transducer is axisymmetric about its axis, the ultrasounds can be simplified into a two-fold finite summation using the Gaussian beam expansion technique. ${ }^{4}$ The source density function of audio virtual sources computed by Eq. (4) is axisymmetric, so the total audio sound of the non-baffled model Eq. (8) and the Green function Eq. (9) can be simplified by integrating the azimuthal angle $\varphi$, yielding

$$
\begin{aligned}
& p_{\mathrm{a}, \mathrm{t}}(\mathbf{r})=2 \mathrm{j} \pi \underset{0}{\underset{\mathrm{a}}{a^{3}}}{ }_{00}^{1} q\left(\mathbf{r}_{\mathrm{v}}\right) G_{0}\left(\mathbf{r}, \mathbf{r}_{\mathrm{v}}\right)\left({\underset{\mathrm{v}}{\mathrm{v}}}^{2}+{ }_{\mathrm{v}}^{2}\right) \mathrm{d}_{\mathrm{v}} \mathrm{d}{ }_{\mathrm{v}}, z<0 \text { or } z>0 \text {, } \\
& G_{0}\left(\mathbf{r}, \mathbf{r}_{\mathrm{v}}\right)=\frac{\mathrm{j} k_{\mathrm{a}}}{2 \pi}{ }_{n=0}\left({ }_{0 n}\left(\mathrm{j} k_{\mathrm{a}} a, \mathrm{j}, \mathrm{j}_{\mathrm{v}}\right) S_{0 n}\left(\mathrm{j} k_{\mathrm{a}} a,\right) S_{0 n}\left(\mathrm{j} k_{\mathrm{a}} a,{ }_{\mathrm{v}}\right) .\right.
\end{aligned}
$$

\section{RESULTS AND DISCUSSIONS}

In this section, both simulation and experiment results are presented. As shown in Fig. 1(b), the experiments were conducted in the hemi-anechoic room in University Technology of Sydney with dimensions of $7.20 \mathrm{~m} \times 5.19 \times 6.77 \mathrm{~m}$ (height) and the PAL used in experiments is a Holosonics Audio Spotlight AS-24i with the surface size of $0.6 \mathrm{~m} \times 0.6 \mathrm{~m}$. In the simulations, a circular piston was driven with a uniform surface vibration velocity amplitude and the radius was set as $0.3385 \mathrm{~m}$ so that its area is the same as that of the rectangular PAL used in experiments, i.e. $0.6^{2} \approx \pi \times$ $0.3385^{2}$.

The relative humidity and the temperature in the experiments were $60 \%$ and $25^{\circ} \mathrm{C}$, respectively. The carrier frequency of the $\mathrm{PAL}$ is $64 \mathrm{kHz}$ measured by a Brüel \& Kjær Type 4939 microphone. All of the aforementioned measured data are set as known parameters in the simulations and the air absorption coefficients are calculated 
according to ISO $9613-1 .{ }^{13}$ The air absorption of audio sounds is neglected to simplify the computation of the spheroidal wave functions that are obtained by modifying the codes of Ref. 10 .

The sound field was measured at a rectangular grid with $60 \times 61=3661$ points in the $x O z$ plane at the height of $1.8 \mathrm{~m}$. In all cases, 60 microphones were located in the $x$ direction from $x=-1.45 \mathrm{~m}$ to $x=1.5 \mathrm{~m}$ with a spacing of $5 \mathrm{~cm}$ and they were measured simultaneously with a customary made 60-channel microphone array. The microphone array was located at 61 different positions in the $z$ direction from $z=-3$ $\mathrm{m}$ to $z=3 \mathrm{~m}$ with a spacing of $10 \mathrm{~cm}$. All the measurement microphones are Brüel $\&$ Kjær Type 4957 calibrated by Brüel \& Kjær 4231 calibrator and the sound pressure at the microphones was sampled with a Brüel \& Kjær PULSE system (the analyzer 3053-B-120 with the front panel UA-2107-120). The fast Fourier transform (FFT) analyzer in PULSE LabShop was used to obtain the FFT spectrum. To avoid the spurious sounds at microphones induced by the intensive ultrasounds radiated by the PAL, all the microphones are covered by a piece of small and thin plastic film ${ }^{14}$. The experimental results (not presented in this paper) show the insertion loss of this plastic film is more than $30 \mathrm{~dB}$ at $64 \mathrm{kHz}$, which is sufficient for blocking the ultrasonic sounds, and less than $0.6 \mathrm{~dB}$ below $1 \mathrm{kHz}$, which is negligible for the audio sound under tests.

Figure 2 shows SPLs of audio sounds along the $z$ axis generated by the finite size disk-shaped PAL using the baffled and non-baffled models and the experimental results. All SPLs in the simulations are normalized to a value so that the SPL at $z=$ $2.0 \mathrm{~m}$ for the baffled PAL is the same as that measured in the experiments. For the audio sounds in the back side $(z<0)$, it cannot be predicted by the baffled model, so there are no data for this model. 


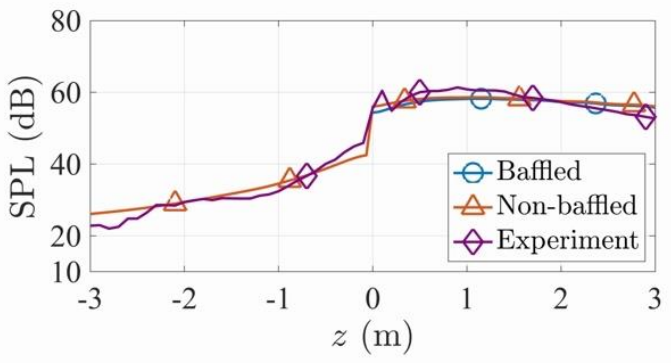

(a)

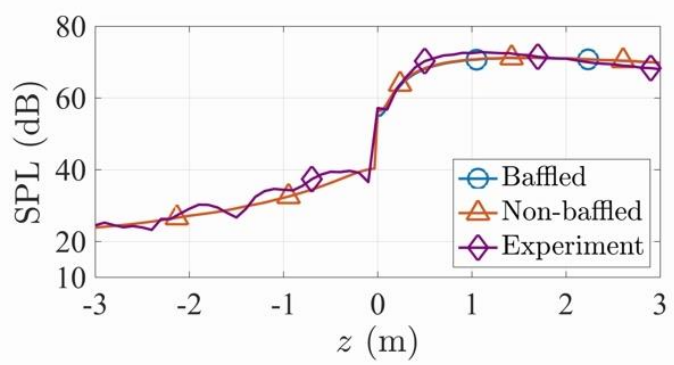

(c)

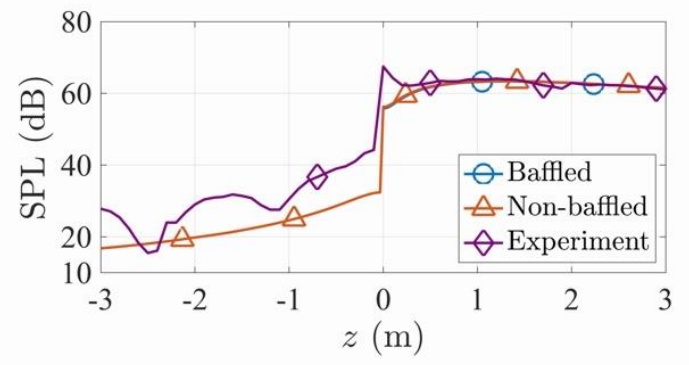

(b)

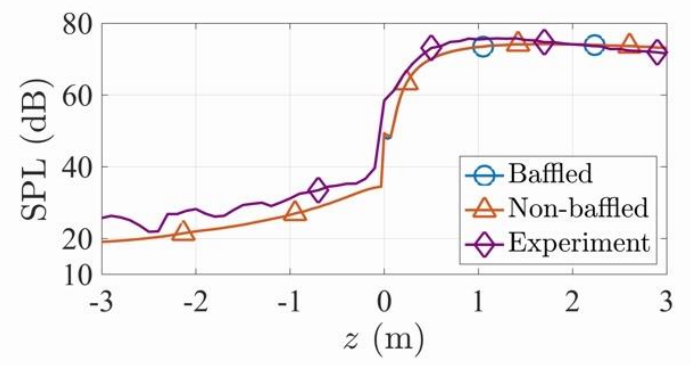

(d)

FIG. 2. (Color Online) SPLs along the $z$ axis at different audio frequencies: (a) 315 $\mathrm{Hz}$, (b) $500 \mathrm{~Hz}$, (c) $800 \mathrm{~Hz}$, and (d) $1 \mathrm{kHz}$, where the curves labeled by "Baffled" and "Non-baffled" are computed with Eqs. (5) and (11), respectively.

It can be found that the values from both the models at all frequencies are almost the same at locations far away in front of the PAL, and the maximum difference is less than $0.4 \mathrm{~dB}$ for $z>1 \mathrm{~m}$. The experimental results in both front and back sides of the PAL are generally in accordance with those predicated by the non-baffled model. Large errors occur at $500 \mathrm{~Hz}$ when $z<0.2 \mathrm{~m}$ which might be caused by the measurement errors, the reflection of grounds, the shape of the PAL, and the scattering effects of the equipment.

The curves of SPL for the non-baffled PAL at $z=-0.1 \mathrm{~m},-0.25 \mathrm{~m},-0.5 \mathrm{~m},-$ $1.0 \mathrm{~m}$, and $-2.0 \mathrm{~m}$ at different frequencies are shown in Fig. 3. The surface SPL of ultrasounds, i.e. the level of $\rho_{0} c_{0}\left|v_{i}\right|, i=1,2$, is set as $125 \mathrm{~dB}$ at all curves for better comparisons. Both Figs. 2 and 3 demonstrate that there are audible audio sounds in the back side of the PAL which is caused by the diffracting of the finite size disk. For example, the SPL is $45 \mathrm{~dB}$ at $z=-0.1 \mathrm{~m}$ at $315 \mathrm{~Hz}$ while the audio sound at $z=1.0$ $\mathrm{m}$ in front of the PAL is about $61.4 \mathrm{~dB}$. 


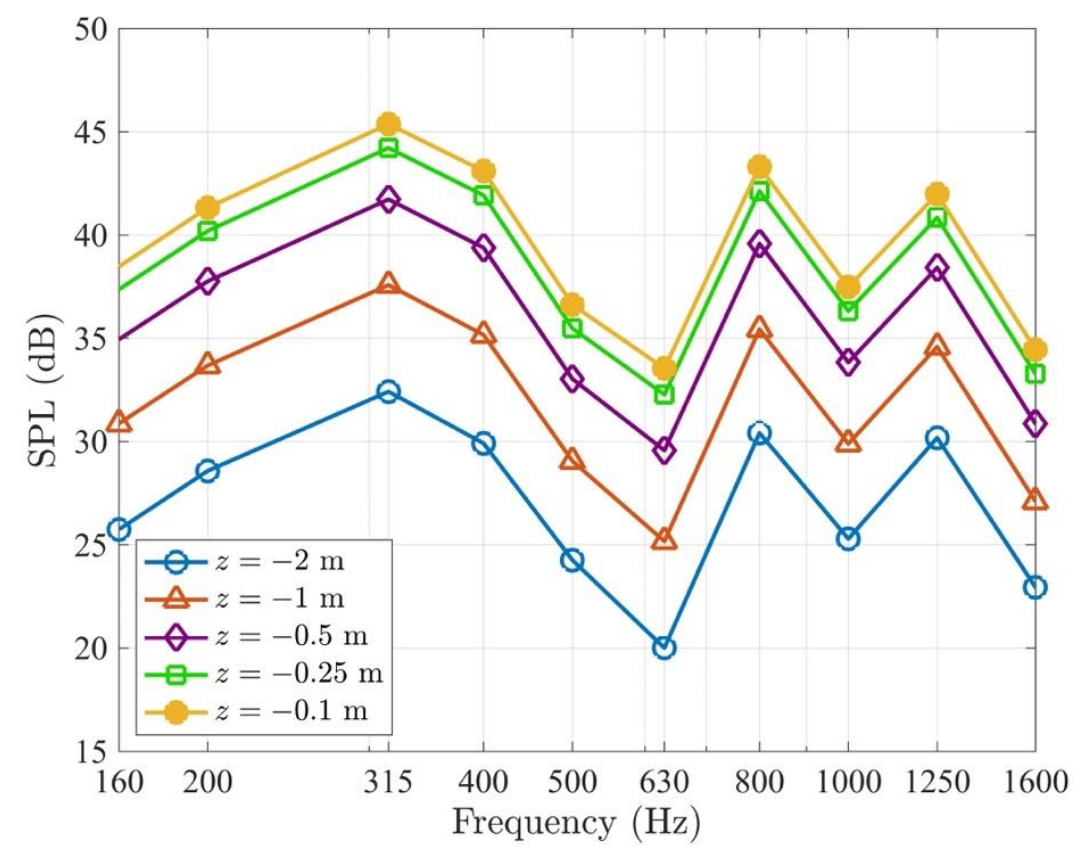

FIG. 3. (Color Online) SPLs along the $z$ axis at $z=-0.1 \mathrm{~m},-0.25 \mathrm{~m},-0.5 \mathrm{~m},-$

$1.0 \mathrm{~m}$, and $-2.0 \mathrm{~m}$ computed with the non-baffled model at 1/3-ocatve center frequencies from $160 \mathrm{~Hz}$ to $1.6 \mathrm{kHz}$ when the surface SPL of ultrasounds is $125 \mathrm{~dB}$.

The SPL is the largest at $315 \mathrm{~Hz}$ (the wavelength is $1.09 \mathrm{~m}$ ) for all cases because the constructive interference of waves is largest for point monopoles when the radius of the PAL is approximately 0.35 times of the wavelength. ${ }^{12}$ As the frequency decreases from $315 \mathrm{~Hz}$, the SPL in the back side decreases due to the fact that the frequency response of the PAL decreases significantly $(12 \mathrm{~dB})$ as the audio frequency is halved. ${ }^{2}$ As the frequency increases from $315 \mathrm{~Hz}$, the SPL in the back side decreases firstly and then reaches the local maximum at $800 \mathrm{~Hz}$ and $1250 \mathrm{~Hz}$ because the diffraction effects are weakened firstly as the wavelength becomes smaller until it reaches other resonant frequencies where the constructive interference of waves is significant.

The SPL distribution in $y O z$ plane at $315 \mathrm{~Hz}$ and $800 \mathrm{~Hz}$ are shown in Fig. 4, where the simulation and experiment results agree well, so the sound fields in the back side of the PAL can be well predicated by the proposed non-baffled model. It is also found that the audio sounds are audible over a large area in the back side of the 
PAL. For example, at $315 \mathrm{~Hz}$, there is an approximately circular region centered at the PAL with the radius of about $1.3 \mathrm{~m}$ where the SPLs are more than $35 \mathrm{~dB}$. Therefore, the effects of the finite size of the PAL should be taken into account.

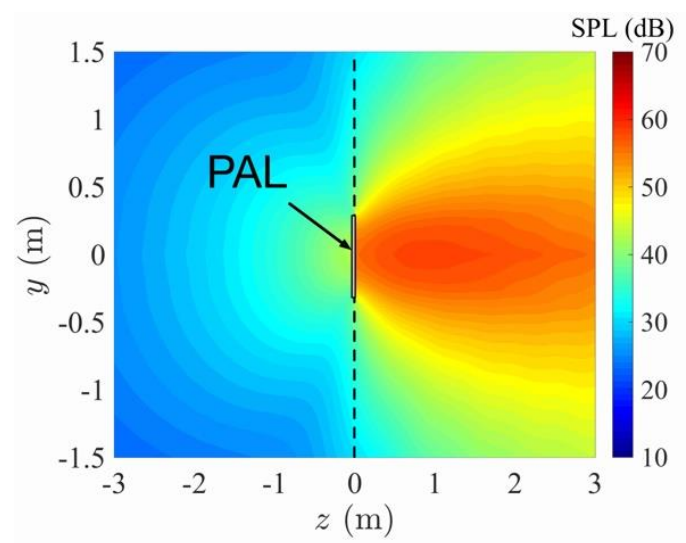

(a)

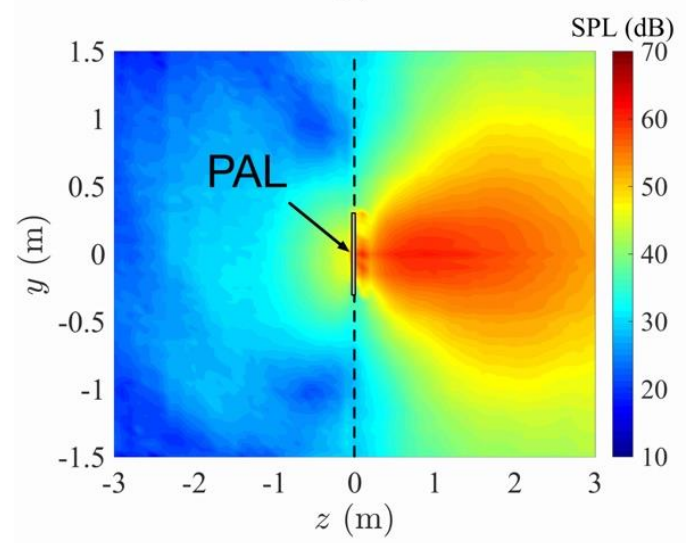

(c)

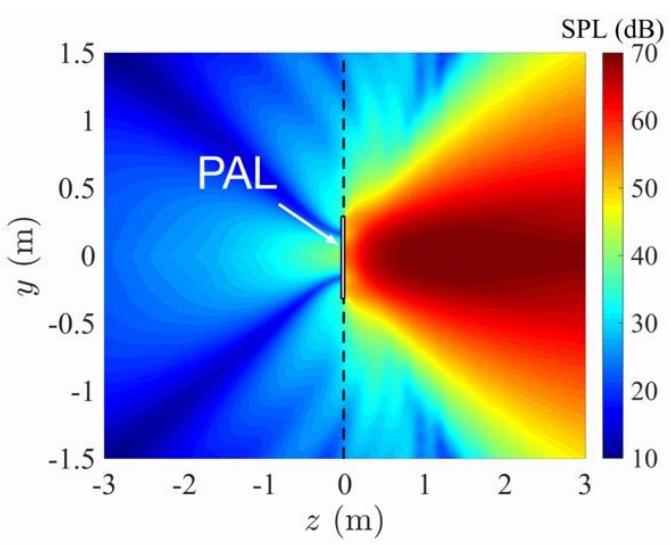

(b)

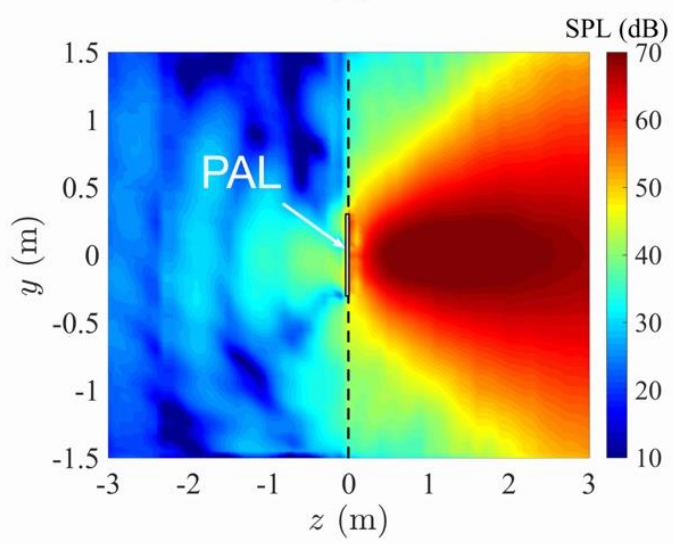

(d)

FIG. 4. (Color Online) The SPLs of audio sounds predicated by the proposed nonbaffled model at (a) $315 \mathrm{~Hz}$ and (b) $800 \mathrm{~Hz}$; and the measured SPLs at (c) $315 \mathrm{~Hz}$ and (d) $800 \mathrm{~Hz}$.

\section{CONCLUSIONS}

A non-paraxial model for the radiation of a non-baffled parametric array loudspeaker in free field is developed based on the quasilinear approximation and the disk scattering theory. In this model, each virtual audio source generated by the ultrasounds in space is regarded as a point monopole and its scattered sound by a finite size disk is computed. Both simulation and experiment results demonstrate that 
audible audio sound exists in the back side of the PAL, indicating that the effects of the finite size of the PAL should be taken into account when calculating the low frequency audio sound field.

\section{ACKNOWLEDGEMENTS}

This research is supported under the Australian Research Council's Linkage Project (No. LP160100616). The authors would like to thank Dr. Shuping Wang at the Centre for Audio, Acoustics and Vibration of UTS for her discussion, comments and suggestions. 


\section{REFERENCES}

1 P. J. Westervelt, "Parametric acoustic array," J. Acoust. Soc. Am. 35(4), 535-537 (1963).

2 M. B. Bennett, and D. T. Blackstock, "Parametric array in air," J. Acoust. Soc. Am. 57(3), 562-568 (1975).

3 W. S. Gan, J. Yang, and T. Kamakura, "A review of parametric acoustic array in air," Appl. Acoust. 73(12), 1211-1219 (2012).

4 M. Červenka, and M. Bednarik, "Non-paraxial model for a parametric acoustic array," J. Acoust. Soc. Am. 134(2), 933-938 (2013).

5 A. Sugahara, H. Lee, S. Sakamoto, and S. Takeoka, "A study on the measurements of the absorption coefficient by using a parametric loudspeaker," INTER-NOISE and NOISE-CON Congress and Conference Proceedings, Hongkong, China, 255(4), 3743-3751 (2017).

6 M. F. Hamilton, and D. T. Blackstock, Nonlinear Acoustics (Acoustical Society of America, New York, 2008).

7 Y. Kagawa, T. Tsuchiya, T. Yamabuchi, H. Kawabe, and T. Fujii, "Finite element simulation of non-linear sound wave propagation," J. Sound Vib. 154(1), 125145 (1992).

8 C. Flammer, Spheroidal Wave Functions (Dover Publications, Mineola, New York, 2014).

9 S. Zhang, and J. Jin, Computation of Special Functions (John Wiley \& Sons, New York, 1996).

${ }^{10}$ A. L. Van Buren, "Accurate calculation of oblate spheroidal wave functions," (2017), arXiv:1708.07929.

${ }^{11}$ H. E. Bass, L. C. Sutherland, A. J. Zuckerwar, D. T. Blackstock, and D. Hester, "Atmospheric absorption of sound: Further developments," J. Acoust. Soc. Am. 97(1), 680-683 (1995).

12 J. Zhong, J. Tao, F. Niu, and X. Qiu, "Effects of a finite size reflecting disk in sound power measurements," Appl. Acoust. 140(1), 24-29 (2018).

${ }^{13}$ ISO 9613-1:1993. Acoustics - Attenuation of sound during propagation outdoors - Part 1: Calculation of the absorption of sound by the atmosphere (International Organization for Standardization, Geneva, 1993).

${ }^{14} \mathrm{P}$. Ji, and J. Yang, "An experimental investigation about parameters' effects on spurious sound in parametric loudspeaker," Appl. Acoust. 148, 67-74 (2019). 